\title{
Batch Microbial Cultures: A Model That Can Account for Environment Changes
}

\author{
Alberto Schiraldi \\ Former Professor of Physical Chemistry at the Department of Food Environment and Nutrition Sciences (DeFENS) of the \\ University of Milan, Milan, Italy \\ Email: alberto.schiraldi@unimi.it
}

How to cite this paper: Schiraldi, A. (2021) Batch Microbial Cultures: A Model That Can Account for Environment Changes. Advances in Microbiology, 11, 630-645. https://doi.org/10.4236/aim.2021.1111046

Received: October 20, 2021

Accepted: November 20, 2021

Published: November 23, 2021

Copyright (C) 2021 by author(s) and Scientific Research Publishing Inc. This work is licensed under the Creative Commons Attribution International License (CC BY 4.0).

http://creativecommons.org/licenses/by/4.0/ (c) (i) Open Access

\begin{abstract}
The so-called growth curve is of help to understand the underlying physiology of microbial cultures. A number of reported models describe the observed growth trends and the effects produced by the changes of the culture environment. However, the collected data (plate counts and/or Optical Density records) very often do not reliably comply with the number of fitting parameters of such models. An alternative semi empirical model describes the observed experimental trends of growth and decay of batch microbial cultures. Major advantages of the model include: reduced number and direct physical meaning of the best-fit parameters, easy comparison between different microbial cultures and/or different environment conditions for a given microbial strain. The experimental data (either plate counts or OD records) allow the estimation of the fitting parameters: that is why the model is substantially empirical and applies to any batch microbial culture. The present paper reports the formal details of the model and its extension to cases of environment changes occurred because of an exterior perturbation. The model seems adequate for predictive microbiology investigations, as well as for studies on the effects of bactericidal drugs.
\end{abstract}

\section{Keywords}

Microbial Cultures, Empirical Model, Environment Changes, Predictive Microbiology

\section{Introduction}

The fundamental role of the so-called growth curve to understand the underlying physiology of microbial cultures received the attention by many authors in the last decades. A number of reported models [1]-[12] describe the observed 
growth trends and the effects produced by the changes of the culture environment, which are of great help in predictive studies [13] aimed to prevent microbial spoilage of food and bio-systems.

Most of them stem from adjustments of the classical logistic model and/or the Gompertz equation for the description of the growth progress: some are dubbed "deterministic" [3] [4], others "stochastic" [5] [6] [7] [8], depending on the relations with the physiology of the microbial population as a whole, or the expected behavior of the single cell [9] [10] [11] [12] [13].

Unfortunately, the data to use (plate counts and/or Optical Density records) demand preliminary treatments, like dilution factors, conversion from OD to population density, and, above all, transfer of the observed values to logarithmic units, that widen the statistical uncertainty of the fitting parameters, which is related to the number of the data and their position along the growth progress [14].

In order to overcome this incongruence, a reduced number of fitting parameters is worth considering. With such an aim, previous works [15] [16] [17] [18] [19] presented a semi-empirical model that described the growth and the decay of batch microbial cultures. Instead of starting from an a priori assumed behavior of the cultures, the model accounts just for the main phenomenological evidence, with no reference to predefined principles, but the behavior of an ideal culture that mimics the trend of the experimental data and can be a reference standard to compare different real batch microbial cultures. The present paper reviews features and potentialities of this model, extending its application to the case of environment changes related to external perturbation or induced by the experimenter. The reported data come from quoted papers where the reader can find the relevant experimental details.

\section{The Model}

The ideal culture of duplicating microbes (extension to other microbial species is straightforward [18]) hosts $N_{o}$ synchronic generation lines stemming from the $N_{o}$ cells of the inoculum, which have the same age. No cell dies during the progress of the growth. The single generation line is a discontinuous succession of steps separated by time intervals that correspond to the duplication time, $\tau$, which changes with the growth progress (Figure 1). Through a suitable function $\tau(t)$, one may replace the discontinuous succession of steps with a continuous trend and describe the progress of the whole microbial population,

$$
N(t)=N_{0} 2^{\frac{t}{\tau(t)}}
$$

The first presentation of the model [15] did not include a formal justification for the choice of the function $\tau(t)$. What follows overcomes this lack of information.

The function $N(t)$ and its time derivative, $\dot{N}=\frac{\mathrm{d} N}{\mathrm{~d} t}$, must fulfill some constraints imposed by the phenomenological evidence: 


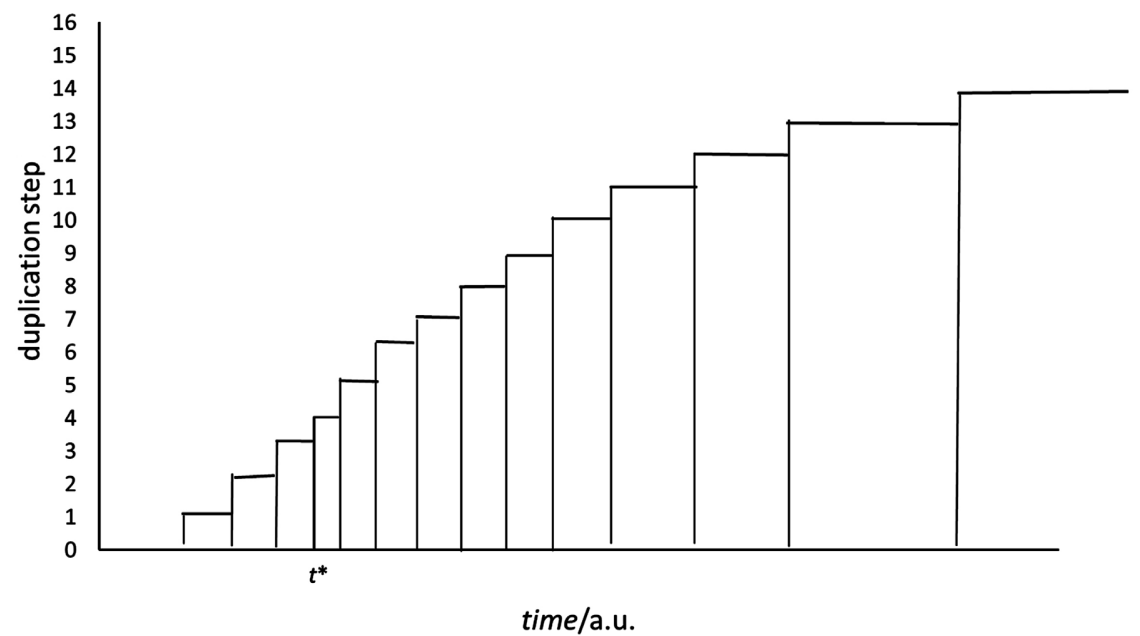

Figure 1. The ideal culture: schematic representation of the duplication steps along one of the $N_{0}$ generation lines stemmed from the $N_{0}$ cells of the inoculum. The variable gap between successive steps reflects the change of the duplication time, $\tau$, with the progress of the growth. All the generation lines progress synchronically. Summing for the $N_{0}$ generation lines and adopting a suitable function $t(t)$, a continuous trend can replace the steps (see text).

1) $\lim _{t \rightarrow \infty} N=N_{\max }=N_{0} 2^{\beta}$ with $\beta=$ constant, which means that $\lim _{t \rightarrow \infty}\left(\frac{t}{\tau}\right)=\beta$

2) $\lim _{t \rightarrow 0} N=N_{0}$ which means that $\lim _{t \rightarrow 0}\left(\frac{t}{\tau}\right)=0$;

3) $\left(\frac{\dot{N}}{N}\right)=\log 2 \frac{\tau-t \dot{\tau}}{\tau^{2}}$ and $\lim _{t \rightarrow \infty}\left(\frac{\dot{N}}{N}\right)=0$ and $\lim _{t \rightarrow 0}\left(\frac{\dot{N}}{N}\right)=0$

4) $\lim _{t \rightarrow 0}\left(\frac{\dot{N}}{N}\right)=\log 2 \lim _{t \rightarrow 0}\left(\frac{1}{\tau}\right) \times \lim _{t \rightarrow 0}\left(1-\frac{t \dot{\tau}}{\tau}\right)=0$

5) $\lim _{t \rightarrow 0}\left(\frac{1}{\tau}\right)=0$; since $\tau>0$, then, $\lim _{t \rightarrow 0} \tau=\infty$ and $\lim _{t \rightarrow 0}\left(1-\frac{t \dot{\tau}}{\tau}\right)=$ constant If $\lim _{t \rightarrow 0} \tau=\frac{\alpha}{t}$, with $\alpha=$ constant, then $\lim _{t \rightarrow 0}\left(1-\frac{t \dot{\tau}}{\tau}\right)=2$, which satisfies the
constraint 5 .

A simple and suitable expression for $t(t)$ therefore is:

$$
\tau(t)=\frac{\alpha}{t}+\frac{t}{\beta}
$$

The parameters $\alpha$ and $\beta$ replace " $a$ " and " $1 / b$ " reported in previous works [15] [16] [17] [18]. The corresponding duplication rate, $1 / \tau$, is null at the extremes $t$ $\rightarrow 0$ and $t \rightarrow \infty$.

Since the parameters $\alpha$ and $\beta$ come from the best fit of the experimental data [15] [16] [17] [18], the model is semi empirical and the function $t(t)$ accounts for every endogenous change of the culture environment (e.g., crowding, $\mathrm{pH}$, produced catabolites, available substrate, etc.) that occurs during the growth progress. The explicit expression of Equation (1) therefore is: 


$$
\log _{2}(N)=\log _{2}\left(N_{0}\right)+\frac{\beta t^{2}}{\alpha \beta+t^{2}}
$$

where $N$ may stand for either population density (CFU/volume unit) or overall number of viable cells (CFU).

According to Equation (3), the largest attainable population density $(t \rightarrow \infty)$ is $N_{0} 2^{\beta}$. This means that $\beta$ is the maximum number of duplication steps undergone by the ideal culture. The ratio $(\beta / \alpha)$ deals with the maximum specific duplication rate (see below).

With the Newton notation for time derivatives, $\dot{N}=\frac{\mathrm{d} N}{\mathrm{~d} t}$ and $\ddot{N}=\frac{\mathrm{d}^{2} N}{\mathrm{~d} t^{2}}$, the specific duplication rate and its acceleration are:

$$
\frac{\dot{N}}{N}=\frac{\mathrm{d} \log _{e}(N)}{\mathrm{d} t}
$$

and

$$
\frac{\mathrm{d}}{\mathrm{d} t}\left[\frac{\mathrm{d} \log _{e}(N)}{\mathrm{d} t}\right]=\frac{\ddot{N} \times N-\dot{N}^{2}}{N^{2}}
$$

At $t=t^{*}$, the specific growth rate attains its maximum, $\mu$, which means that its acceleration is null,

$$
\left(\frac{\ddot{N} \times N-\dot{N}^{2}}{N^{2}}\right)_{t=t^{*}}=0,
$$

which yields

$$
\left(\ddot{N} \times N-\dot{N}^{2}\right)_{t=t^{*}}=0
$$

From Equation (3), one gets:

$$
\begin{gathered}
\log _{e}(N)=\log _{e}\left(N_{0}\right)+\log _{e}(2) \times \frac{\beta t^{2}}{\alpha \beta+t^{2}} \\
\frac{\dot{N}}{N}=\log _{e}(2) \times \frac{2 \alpha \beta^{2} t}{\left(\alpha \beta+t^{2}\right)^{2}}
\end{gathered}
$$

and

$$
\begin{gathered}
\dot{N}=N \times \log _{e}(2) \times \frac{2 \alpha \beta^{2} t}{\left(\alpha \beta+t^{2}\right)^{2}} \\
\ddot{N}=\log _{e}(2) \times\left[\dot{N} \times \frac{2 \alpha \beta^{2} t}{\left(\alpha \beta+t^{2}\right)^{2}}+N \times \frac{2 \alpha \beta^{2}\left(\alpha \beta+t^{2}\right)^{2}-8 \alpha \beta t^{2}\left(\alpha \beta+t^{2}\right)}{\left(\alpha \beta+t^{2}\right)^{4}}\right]
\end{gathered}
$$

Introducing the last two expressions in Equation (5), one gets

$$
\begin{aligned}
\left(\ddot{N} N-\dot{N}^{2}\right)_{t=t^{*}}= & {\left[\log _{e}(2)\right]^{2} \times N^{* 2}\left[\frac{2 \alpha \beta^{2} t}{\left(\alpha \beta+t^{2}\right)^{2}} \times \frac{2 \alpha \beta^{2} t}{\left(\alpha \beta+t^{2}\right)^{2}}\right.} \\
& \left.+\frac{2 \alpha \beta^{2}\left(\alpha \beta+t^{2}\right)^{2}-8 \alpha \beta t^{2}\left(\alpha \beta+t^{2}\right)}{\left(\alpha \beta+t^{2}\right)^{4}}-\frac{4 \alpha^{2} \beta^{4} t^{2}}{\left(\alpha \beta+t^{2}\right)^{4}}\right]_{t=t^{*}} \\
= & 0
\end{aligned}
$$


which yields:

$$
\alpha^{2}-3 \frac{t^{*^{4}}}{\beta^{2}}-2 \alpha \frac{t^{*^{2}}}{\beta}=0
$$

Replacing $\left(t^{\star}\right)^{2}$ with $x$ and solving the equation

$$
\alpha^{2}-3 \frac{x^{2}}{\beta^{2}}-2 \alpha \frac{x}{\beta}=0 \text { or, } x^{2}+2 \frac{\alpha \beta}{3} x-\frac{\alpha^{2} \beta^{2}}{3}=0
$$

one finally gets $x=\left(t^{*}\right)^{2}=\frac{\alpha \beta}{3}$, which means that,

$$
\begin{gathered}
t^{*}=\sqrt{\frac{\alpha \beta}{3}} \\
N\left(t=t^{*}\right)=N^{*}=N_{0} 2^{\beta / 4} \\
\left(\frac{\dot{N}}{N}\right)_{t=t^{*}}=\mu=\log _{e}(2) \frac{3 \sqrt{3}}{8} \sqrt{\frac{\beta}{\alpha}}
\end{gathered}
$$

The straight-line tangent to the growth curve at $t=t^{*}$ is

$$
\log _{e}\left(\frac{N}{N_{0}}\right)=\mu t-\log _{e}(2) \frac{\beta}{8}
$$

or

$$
\log _{2}\left(\frac{N}{N_{0}}\right)=\frac{3 \sqrt{3}}{8} \sqrt{\frac{\beta}{\alpha}} t-\frac{\beta}{8}
$$

For $N=N_{0}$, this straight line crosses the $t$-axis at

$$
t(0)=\frac{1}{3} \sqrt{\frac{\alpha \beta}{3}}=\frac{t^{*}}{3}
$$

and reaches the value $N=N_{0} 2^{\beta}$ at

$$
t=t_{\text {end }}=3 t^{*}
$$

At $t_{\text {end }}$ the microbial population reaches the value

$$
\log _{2}\left(\frac{N}{N_{0}}\right)_{t=t_{\text {end }}}=\frac{3}{4} \beta
$$

The above equations do not include the no-growth period that often precedes the onset tail of the growth curve (several $\log \left(N / N_{0}\right) \approx 0$ experimental data). The model accounts for the duration of such latency gap with a time shift, $t_{0}$, to subtract from the value of $t$ in the above relationships. With this additional condition, the expected trend of the growth curve of the ideal population looks like the full line shown in Figure 2, where the use of $\log _{2}$ scale is for the sake of a clear picture.

Figure 2 shows that the straight-line tangent to the growth trend at $t^{*}$ has an intercept, $\gamma$, for $t=0$.

When $t_{0}=0, \gamma=-\beta / 8$. Its cross points with the levels $\log _{2}\left(N / N_{0}\right)=0$ and $\log _{2}\left(N_{\max } / N_{0}\right)$ occur at $t(0)=\left[t_{0}+\left(t^{*}-t_{0}\right) / 3\right]$ and $t_{\text {end }}=\left[t_{0}+3\left(t^{*}-t_{0}\right)\right]$, 


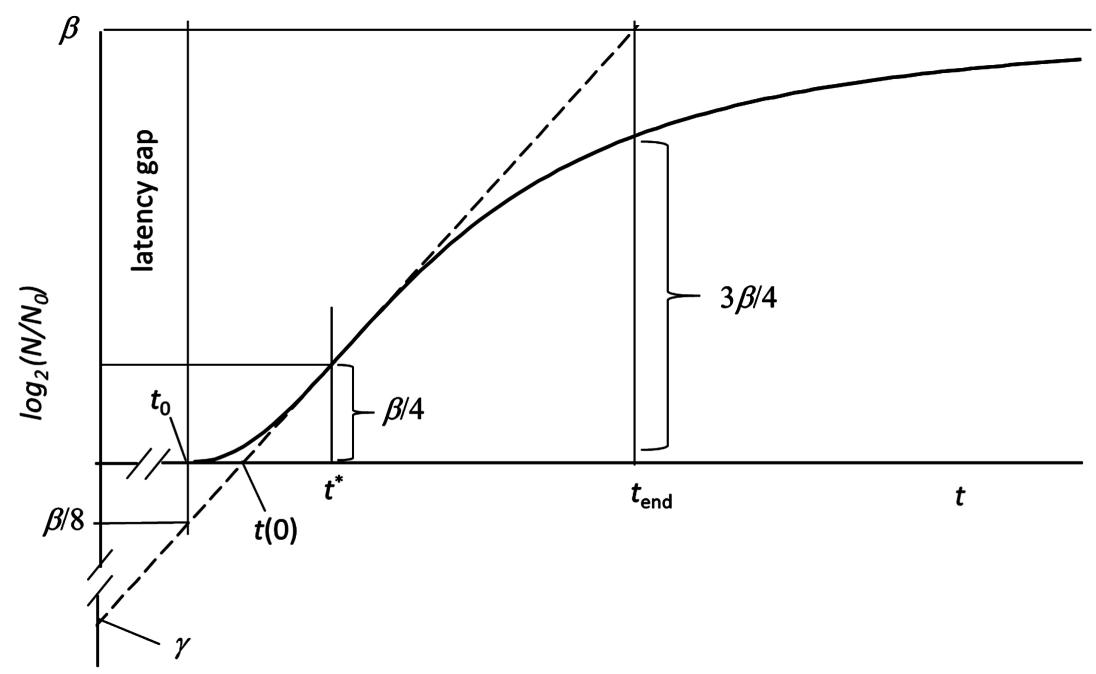

Figure 2. Sketch of the ideal growth curve according to the proposed model.

respectively. The lag phase, $\lambda$, reported in the literature [20] [21] [22] [23] corresponds to $\left[t(0)-t_{0}\right]$, namely, the time encompassed by the onset tail of the growth curve.

The model allows a representation in reduced units, $\xi=\frac{\log _{2}\left(\frac{N}{N_{0}}\right)}{\beta}$ and $t_{R}=\left(t-t_{0}\right) /\left(t^{*}-t_{0}\right)$, that can gather the growth curves of different cultures, or those observed for a given culture in different environments, in a single master plot [15] [16] [17] [18] (Figure 3).

The model was successfully applied to a number of batch cultures [15] [16] [17] [18] and, because of its semi-empirical nature, it actually seems of general validity. What is more, a recent paper [24] reports a comparison with other approaches and shows its better reliability.

The crucial parameters of the model are $t^{*}$ and $\mu$, which identify the condition of enough biochemical power of the cells to sustain the maximum duplication rate. Since ATP is the most used compound in the cytoplasmic processes, one expects that ATP should reach the maximum concentration at $t=t^{*}$. An example comes from the data (unpublished) of an old work [25] of the present author dealing with the culture of microbes responsible for the spoilage of historical marbles (Figure 4). Although the overall ATP content is proportional to the population density, the ATP content per cell occurs just when the population attains the maximum specific growth rate.

While the parameters $\alpha$ and $\beta$ of the model have a clear biological and physical meaning (see above), the parameter $\gamma$ remained rather obscure (in spite of some tentative explanation [17]) until the improvement achieved with a suitable choice of the time origin [19].

\section{The Issue of the Time Origin}

The above description implies that the origin of the time scale of the experimenter 


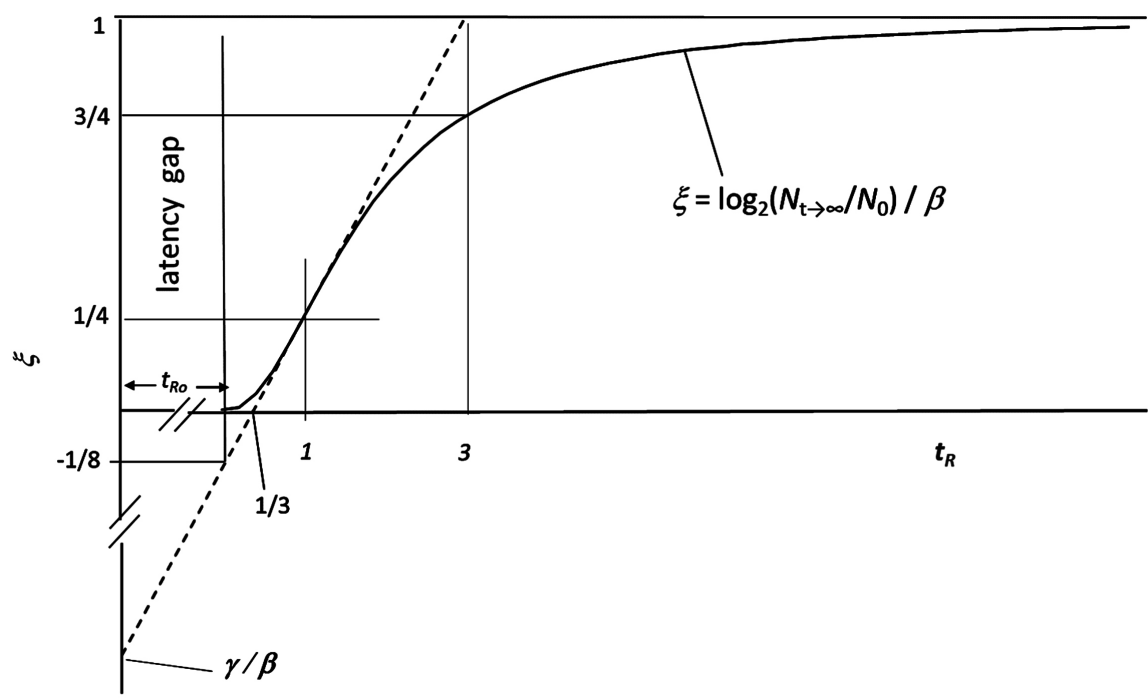

Figure 3. Master plot of growth curves with different $\alpha, \beta$ and $t_{0}$ parameters. Such a plot can gather the growth curves of all the duplicating microbes in any medium and environment, provided the relevant changes are just those related to the growth process itself, with no external perturbation.

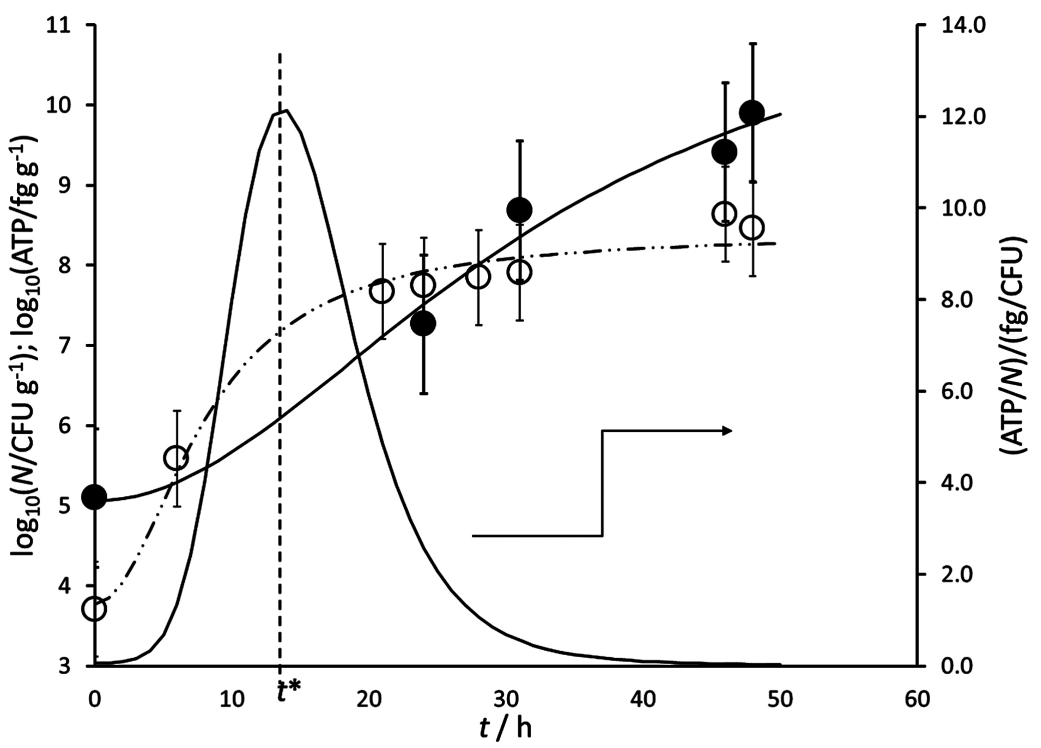

Figure 4. Plate count data (full circles) and ATP content (open circles) of microbes (Bacillus genera) found on the marbles of Ca' d'Oro (Venice, Italy). For the experimental details, see [25]. The peak function directly comes from the fitting curves and reproduces the ATP content per cell.

may coincide with the time scale of the microbial culture: which is not necessarily the case. Although usually neglected or left to an arbitrary choice (as in the tests suggested in the ComBase data bank), this is a major issue to face.

It was suggested [19] that a reasonable choice for the origin of the time scale of the microbial culture, $t_{\text {start }}$, could be the intercept of the straight line tangent to the growth curve at $t^{*}$ with the level $\log (N / \mathrm{CFU})=0$, i.e., $N=1 \mathrm{CFU}$, no matter the base for the logarithm (Figure 5). The time of the microbial culture, $\theta$, 


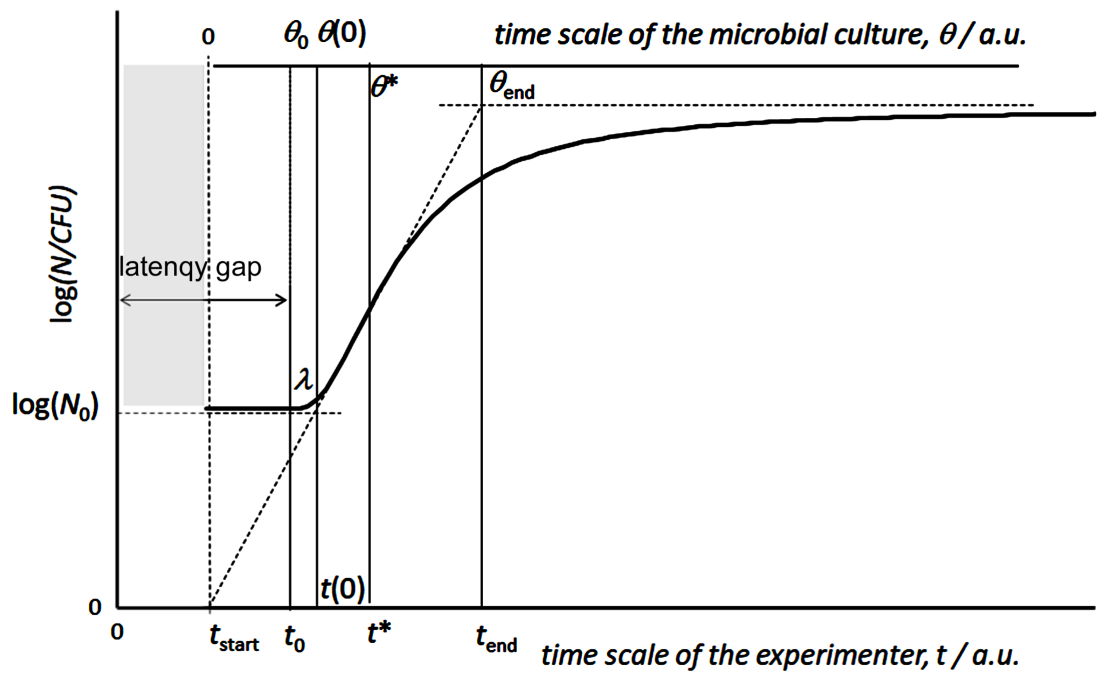

Figure 5. The origin, $t_{\text {start }}$, of the time scale of the microbial culture (continuous heavy line) is singled out by extrapolating the straight line that corresponds to the maximum specific growth rate to $\log (N)=0$, no matter the base of the logarithm. The shaded region corresponds to biological activity not aimed at cell duplication.

therefore corresponds to $\left(t-t_{\text {start }}\right)$, and the critical thresholds are accordingly scaled: $\theta_{0}=\left(t_{0}-t_{\text {start }}\right), \theta^{*}=\left(t^{*}-t_{\text {start }}\right)$, etc.

Data at $\theta<0$ would reflect cellular activities not aimed at the duplication, while those observed for $0<\theta<\theta_{0}$ correspond to cellular activities aimed at preparing the growth onset of the microbial culture. This choice of the time origin is consistent with the assumption that, in a given environment and at a given temperature, the maximum specific duplication rate, $\mu$, and the maximum achievable population density, $N_{\max }$, do not depend on the starting population density, $N_{0}$, being intrinsic peculiarities of the system [10]. One may therefore rigidly shift the observed growth trends along the time axis, so as to align them on the same straight line that starts at $\theta=0$, has a slope equal to $\mu$, and goes through the $\theta^{*}$ 's of the trends considered (Figure 6).

As shown in Figure 6, for a given $N_{\max }$, the lower $\log \left(N_{0}\right)$, the shorter the corresponding latency gap, $\theta_{0}$, which precedes the onset tail of the growth trend: are cultures with a larger population density more reluctant to undertake duplication? Cell crowding would likely adverse a further growth. An opposite trend seems that of the $\left[\theta(0)-\theta_{0}\right]$ time span, that corresponds to the lag-phase $\lambda$ reported in the literature [20] [21] [22] [23]: it decreases for increasing $N_{0}$. It seems that $\theta_{0}$, rather than $\lambda$, could reflect the early adjustments [10] [23] that prepare the cells to attain the maximum specific duplication rate.

When $t_{\text {start }}$ is negative in the scale of the experimenter, the observed growth trend can miss a large part of the no-growth latency phase, giving the fake view of a steep onset of the growth progress [19]. This is more likely a failure of the experimental design (e.g., the inoculum contains already fast duplicating cells, or the time span between observations is too wide, etc.), rather than a peculiarity of the microbial physiology. 


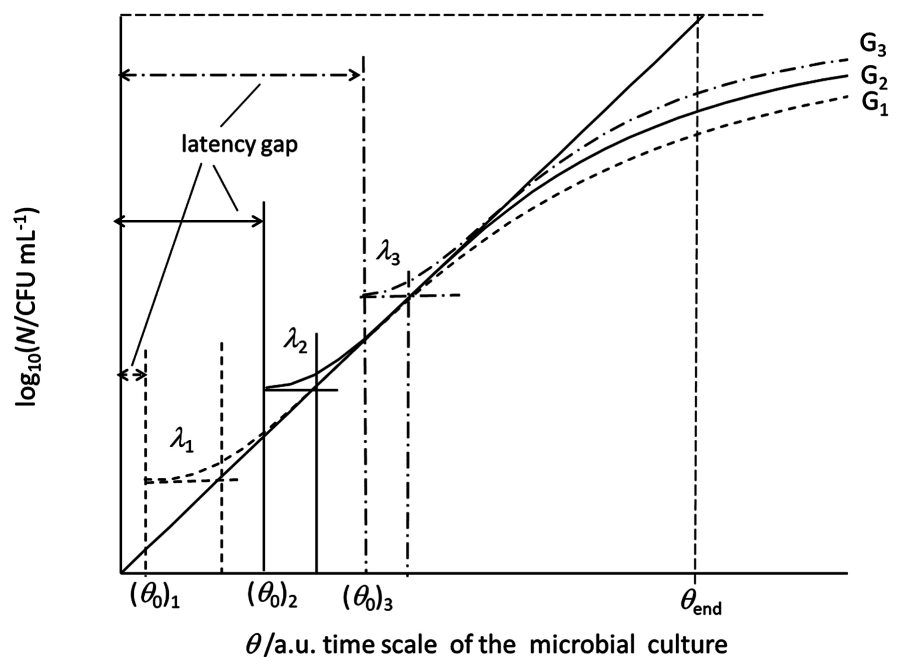

Figure 6. Rigid shifts of growth trends starting from different $N_{0}$ levels allow their alignment on the same straight line that has a slope equal to the maximum specific rate and goes through their respective $\theta^{*} . \theta_{0}$ is the duration of the latency gap that precedes the onset of the growth. The $\left[\theta(0)-\theta_{0}\right]$ intervals correspond to the duration of the so-called [20] [21] [22] [23] lag phase, $\lambda$. For some case studies, the reader is addressed to ref. [19].

If $N$ is the population density (instead of the total number of viable cells), the intercept to consider for $t_{\text {start }}$ still is at $\log [N /$ (CFU/volume units) $]=0$, no matter the volume units. This choice makes sense if referred to the condition of a single cell in the overall volume of the culture, $V$ (which is supposed to remain substantially unchanged during the growth progress). In other words, a single cell in the overall volume, $V$, would produce $\left(N_{0} \times V\right)$ cells, i.e., the real starting population density, in $\theta_{0}$ time units, were it able to duplicate at a pace equal to $\mu$. This choice of the time scale was applied to batch cultures of Aeromonas hydrophila, Yersinia enterocolitica, and Listeria monocytogenes [19].

When only OD data are available and neither $N_{0}$ nor $N_{\max }$ can be separately determined with adequate accuracy, the ratio $\left(N / N_{0}\right) \approx\left(\mathrm{OD} / \mathrm{OD}_{0}\right)$ is the most reliable correlation with the cell density. Consequently, $t_{\text {start }}$ and the split of the latency phase in growth promoting and non-promoting sub spans are not achievable. The only assessable time threshold is $t_{0}$, which can be either positive or negative in the experimenter's time scale.

\section{The Population Decay}

Once the microbial culture achieves the maximum population density, it enters in a steady phase, before undertaking a decay process. The trend of the experimental data suggests an estimation of the duration of the steady phase (which is otherwise unpredictable) and the start of the decline. Putting the start of the decline as the origin of the time scale, $\theta=0$, the function $\exp \left(-\theta^{2} / d\right)$, where $d$ is a constant determined empirically, can reliably fit [17] the population decay that occurs with a steeply increasing rate. The trend of this function shows an inflection point in the $N-v s-\theta$ plot, but no inflection in the $\log (N)-v s-\theta$ plot (Figure 7). Deviations from such a trend concern only microbial species that are 


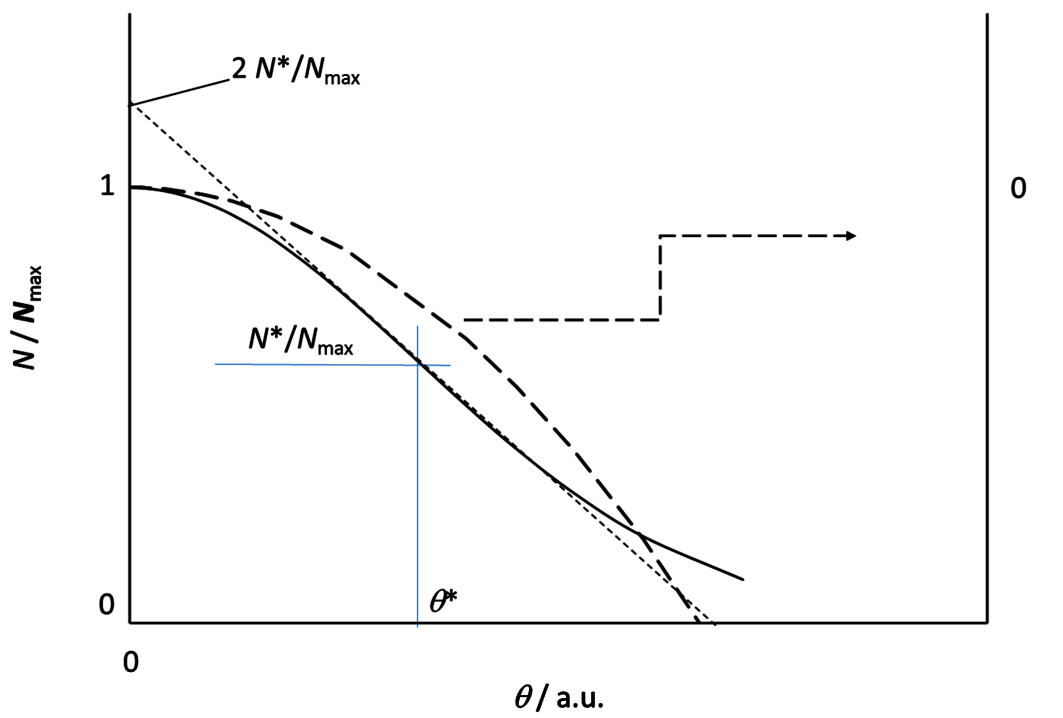

Figure 7. Decay trend according to the model [17]. The origin of the time scale is the start of the decline determined from the trend of the experimental data.

able to modify their own metabolism, or undergo sporulation, to contrast the adverse environment [17].

Figure 8 shows the case of a microbial culture that decays after a long steady condition that follows the growth rising trend.

However, microbial cultures that grow in an adverse medium since the start of the growth often do not show any steady plateau [17] [27], but go through a broad maximum that occurs before the attainment of the "potential" maximum capacity of the system, $N_{0} 2^{\beta}$, assessed by applying the model only to the data of the ascending trend. The best fit of such evolution of the microbial culture comes from a combination of the expressions for the rising and declining trends, with reference to their common value, namely, $N_{\max }$, and a common time scale with the zero point at the onset of the growth [17],

$$
N=N_{\max } \times 2^{-\frac{\alpha \beta^{2}}{\alpha \beta+t^{2}}} \times \exp \left[-\frac{t^{2}}{d}\right]
$$

Since the model assumes that no cell death occurs before the attainment of $N_{\max }$, one can obtain the effect of the eventual decline by selecting large $d$ values (i.e., negligible decay rate in the time range of prevailing growth; this approach is used also in the fit reported in Figure 8). Figure 9 shows the case of a culture of Listeria monocytogenes/innocua [28] that goes through a broad maximum of the population density, not showing a real intermediate plateau between growth and decline. Equation (21) fits the whole trend (growth + decay).

\section{Environment Changes Induced by the Experimenter}

Although the present model implies only changes of the environment related to the growth progress and accounted for by the function $\tau(t)$, it can still be of help when changes, like changes of temperature, $\mathrm{pH}$, water activity, or concentration 

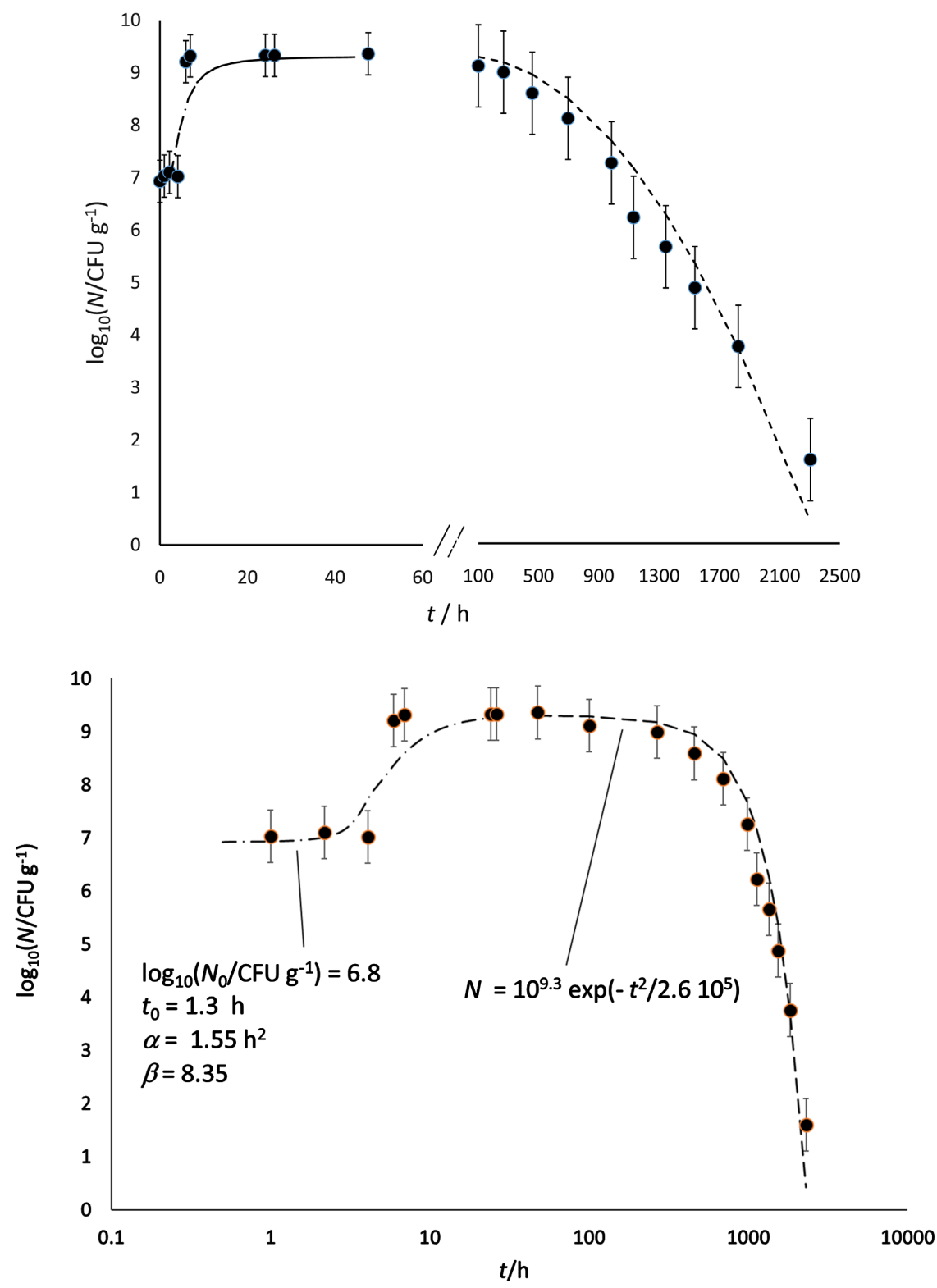

Figure 8. Escherichia coli in feta cheese at $15^{\circ} \mathrm{C}$ and starting $\mathrm{pH}=6.75$. Data after [26]. On the right side, the variable $t$ is in $\log _{10}$ scale for a better view. The fitting parameters of both the rising and the declining trend refer to the same time scale: that is why the parameter " $d$ " (see text) has such large value of $\left(2.6 \times 10^{5} \mathrm{~h}^{2}\right)$.

of some critical substrate, are induced by the experimenter or an external perturbation. Any change of these modifies the growth trend, which nonetheless must comply with some constraints when passing from environment 1 to environment 2:

$$
\begin{gathered}
\theta_{01}=\frac{\log _{2}\left(N_{0}\right)}{\text { slope }_{1}}-\frac{1}{8} \frac{\beta_{1}}{\text { slope }_{1}} \\
\theta_{02}-(\theta=0)_{2}=\frac{\log _{2}\left(N_{\text {change }}>N_{0}\right)}{\text { slope }_{2}}-\frac{1}{8} \frac{\beta_{2}}{\text { slope }_{2}}
\end{gathered}
$$




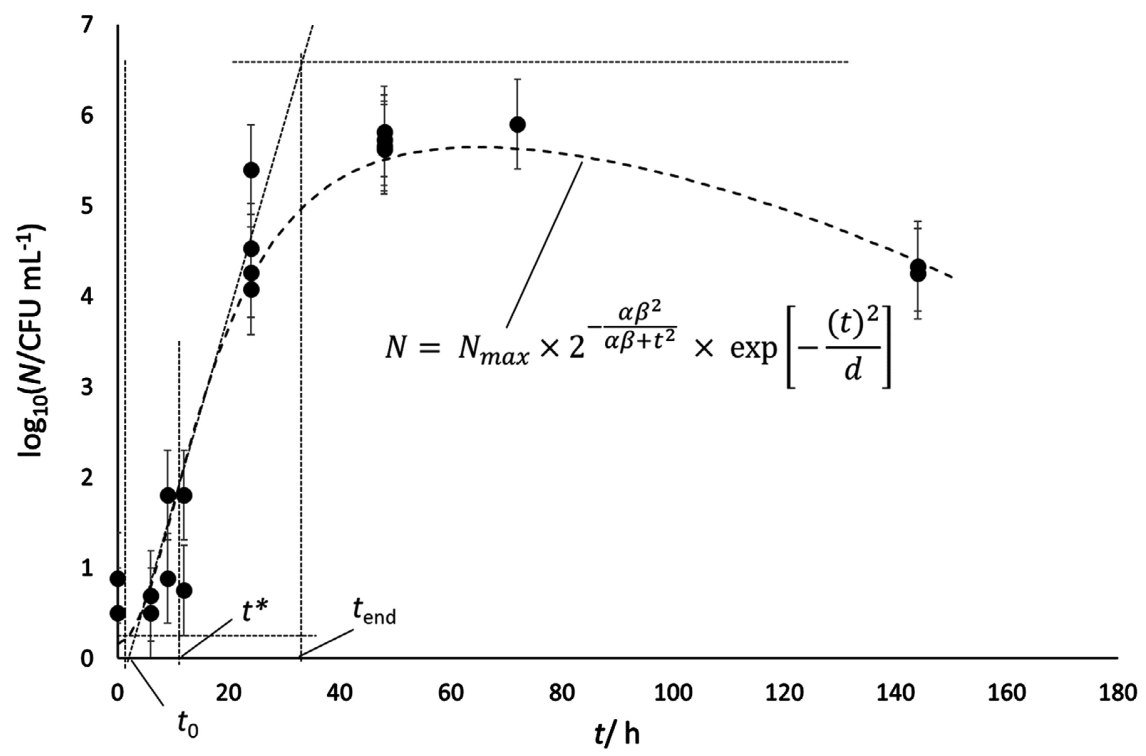

Figure 9. Listeria monocytogenes/innocua in milk shake at $25^{\circ} \mathrm{C}$. Data from ComBase: ID:Lm_Nat_25C_T3, after [28]. The values of the best-fit parameters are: $\log _{10}\left(N_{\max } / \mathrm{CFU}\right.$ $\left.\mathrm{mL}^{-1}\right)=6.5 ; \alpha=15 \mathrm{~h}^{2}, \beta=21, d=4452 \mathrm{~h}$.

where "slope" stands for $\frac{3 \sqrt{3}}{8} \sqrt{\frac{\beta}{\alpha}}$ (see Equation (17)). The level $N_{\text {change, }}$ attained at the environment change, becomes the new starting level (after some oscillation [20] [29]). Because of the environmental change, the time origin too changes (Equation (23)). The new time origin, $(\theta=0)_{2}$, is unpredictable and comes a posteriori from the experimental evidence of the new growth trend. The straight line corresponding to the new maximum specific rate crosses the level $\left[\log _{2}\left(N_{\text {change }}\right)-1 / 8 \beta_{2}\right]$ at $\theta_{02}$ (Figure 10).

Examples of such environment changes induced by the experimenter were so far proposed ([20] and therein quoted papers).

Finally, the environment 2 could be strongly adverse to the growth progress and imply the death of the cells, as in the case of injection of a bactericidal drug. In such a case, one should observe the decline of the microbial population. A steady phase may or may not occur before the decline.

When a deathly change hits the environment of the culture, the growth trend would first relent because of the death of the old (weakest) cells. Once death involves the younger generations, which represent increasingly larger fractions of the population, an overall decay of the population density takes place. This is just the cascade trend observed experimentally. Figure 11 shows the expected declining trend and two possible "connections" with the preceding rising trend.

The growth curve will likely show just a modest downward bend when the environment changes and undergo an increasingly faster decline later on. Replacing $\left(N_{0} 2^{\beta}\right)$ with $N_{\text {change, }}$, the same fitting equation applies also to cases of interruption of the growth trend because of an abrupt environment change induced by the experimenter or an external perturbation. 

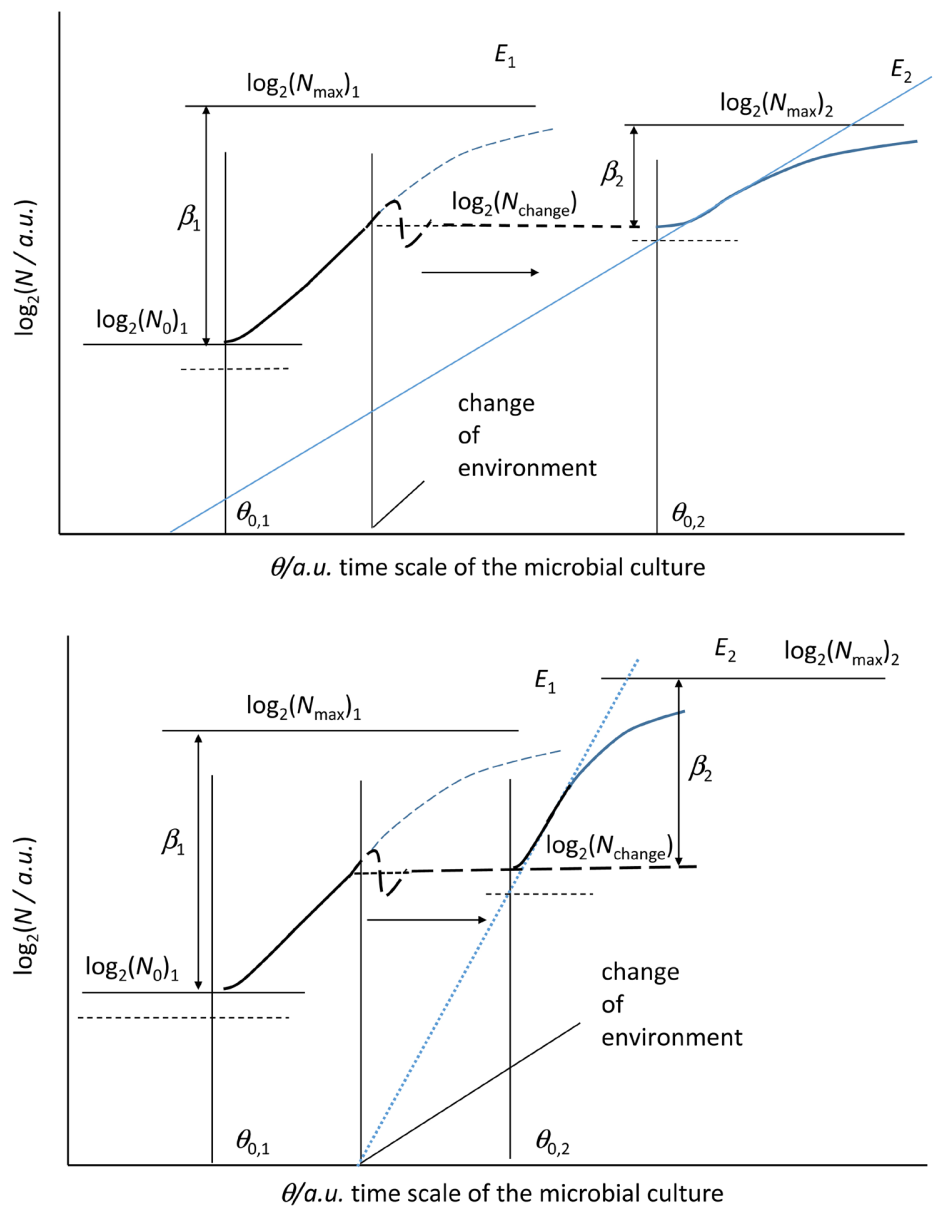

Figure 10. The growth trend interrupted because of an abrupt change of the environment induced by the experimenter or an external perturbation. The change worsens (left) or enhances (right) the growth progress. The light horizontal lines beneath the $\log \left(N_{0}\right)$ and $\log \left(N_{\text {change }}\right)$ levels correspond to $1 / 8$ of the $\log \left(N_{\max } / N_{0}\right)$ and $1 / 8$ of the $\log \left(N_{\text {max }} / N_{\text {change }}\right)$ gap and allow identification of the corresponding $\theta_{0}$ values.

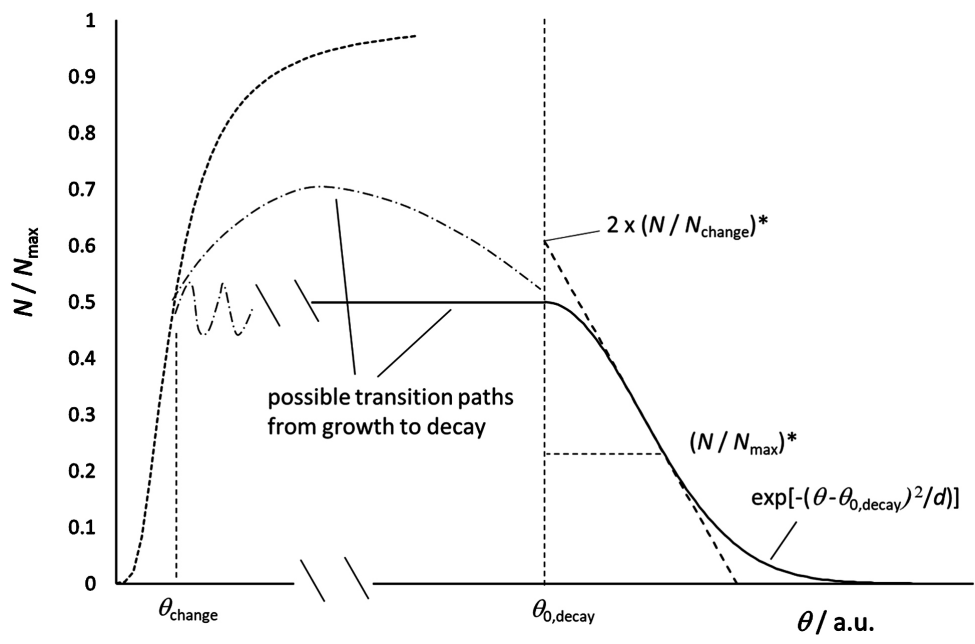

Figure 11. The environment change causes the decline of the cell density. The decline

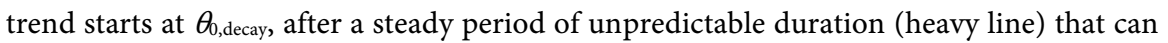
also appear as a broad maximum (light dashed line) between rising and declining trend. 


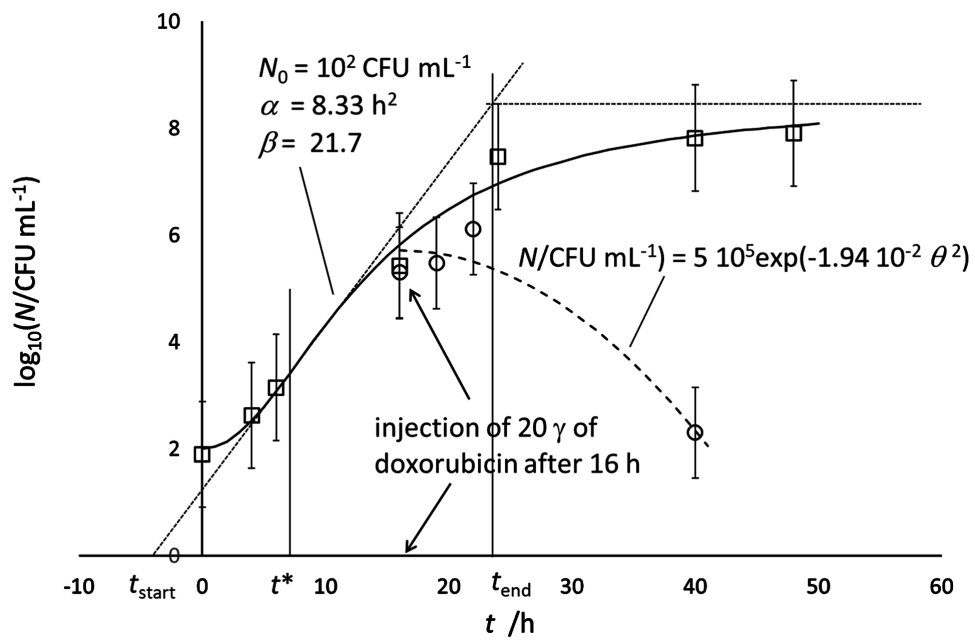

Figure 12. The unperturbed growth of L. helveticus (open squares and continuous line). The inoculation of a $20 \gamma\left(\gamma=1 \mu \mathrm{g} \cdot \mathrm{mL}^{-1}\right)$ dose of doxorubicin after $16 \mathrm{~h}$ growth produces the decline of the microbial population (open circles and dotted line). Data after [30]. In the inserted equation, $\theta$ stands for $(t-16 \mathrm{~h})$. Notice that $t_{\text {start }}$ is negative.

$$
N=N_{\text {change }} \exp \left(-\frac{\theta^{2}}{d}\right)
$$

The effect of a bactericidal drug, like doxorubicin, added in the course of the growth of Lactobacillus helveticus ATCC (American Type Culture Collection) $15009 \mathrm{~T} L$ in MRS broth at $37^{\circ} \mathrm{C}$ (Figure 12) is an example of environment change induced by the experimenter (for experimental details see [30]).

\section{Conclusions}

A semi empirical model describes the observed experimental trends of growth and decay of batch microbial cultures. It also applies to cases of environment changes induced by the experimenter, like inoculation of a bactericidal drug, or anyway occurred because of exterior perturbation. Major advantages of the model include: reduced number and direct physical meaning of the best-fit parameters, easy comparison between different microbial cultures and/or different environment conditions for a given microbial strain. The experimental data (either plate counts or OD records) allow the estimation of the fitting parameters: that is why the model is substantially empirical and applies to any batch microbial culture.

The model seems adequate for predictive microbiology investigations, as well as for studies on the effects of bactericidal drugs.

\section{Conflicts of Interest}

The author declares no conflicts of interest regarding the publication of this paper.

\section{References}

[1] Campbell, A. (1957) Synchronization of Cell Division. Bacteriological Reviews, 21, 
263-272. https://doi.org/10.1128/br.21.4.263-272.1957

[2] Ingraham, J.L., Maaløe, O. and Neidhardt, F.C. (1983) Growth of the Bacterial Cells. Sinauer Associate, Sunderland.

[3] Zwietering, M.H., Jongenburger, I., Rombouts, F.M. and van't Riet, K. (1990) Modeling of the Bacterial Growth Curve. Applied and Environmental Microbiology, 56, 1875-1881. https://doi.org/10.1128/aem.56.6.1875-1881.1990

[4] Baranyi, J. and Roberts, T.A. (1994) A Dynamic Approach to Predicting Bacterial Growth in Food. International Journal of Food Microbiology, 23, 277-294.

https://doi.org/10.1016/0168-1605(94)90157-0

[5] Baranyi, J. (1998) Comparison of Stochastic. Comparison of Stochastic and Deterministic Concepts of Bacterial Lag. Journal of Theoretical Biology, 192, 403-408. https://doi.org/10.1006/jtbi.1998.0673

[6] Baranyi, J. and Pin, C. (1999) Estimating Bacterial Growth Parameters by Means of Detection Times. Applied and Environmental Microbiology, 65, 732-736. https://doi.org/10.1128/AEM.65.2.732-736.1999

[7] Neidhardt, F.C. (1999) Bacterial Growth: Constant Obsession with $\mathrm{d} N / \mathrm{d} t$. Journal of Bacteriology, 181, 7405-7408. https://doi.org/10.1128/JB.181.24.7405-7408.1999

[8] Baranyi, J. and Pin, C. (2001) A Parallel Study on Bacterial Growth and Inactivation. Journal of Theoretical Biology, 210, 327-336.

https://doi.org/10.1006/jtbi.2001.2312

[9] Kutalik, Z., Razaz, M. and Baranyi, J. (2005) Connection between Stochastic and Deterministic Modelling of Microbial Growth. Journal of Theoretical Biology, 232, 285-299. https://doi.org/10.1016/j.jtbi.2004.08.013

[10] Schaechter, M. (2006) From Growth Physiology to Systems Biology. International Microbiology, 9, 157-161.

[11] Scott, M. and Hwa, T. (2011) Bacterial Growth Laws and Their Applications. Current Opinion in Biotechnology, 22, 559-565. https://doi.org/10.1016/j.copbio.2011.04.014

[12] Egli, T. (2015) Microbial Growth and Physiology: A Call for Better Craftsmanship. Frontiers in Microbiology, 6, 287. https://doi.org/10.3389/fmicb.2015.00287

[13] Stavropoulou, E. and Bezirtzoglou, E. (2019) Predictive Modeling of Microbial Behavior in Food. Foods, 8, 654. https://doi.org/10.3390/foods8120654

[14] Poschet, F., Bernaerts, K., Geeraerd, A.H., Scheerlink, N., Nicola1, J.F. and Van Impe, J.F. (2004) Sensitivity Analysis of Microbial Growth Parameter Distributions with Respect of Data Quality and Quantity by Using Monte Carlo Analysis. Mathematics and Computers in Simulation, 65, 231-243.

https://doi.org/10.1016/j.matcom.2003.12.002

[15] Schiraldi, A. (2017) Microbial Growth in Planktonic Conditions. Cell \& Developmental Biology, 6, 185.

[16] Schiraldi, A. (2017) A Self-Consistent Approach to the Lag Phase of Planktonic Microbial Cultures. Single Cell Biology, 6, 3.

[17] Schiraldi, A. (2020) Growth and Decay of a Planktonic Microbial Culture. International Journal of Microbiology, 2020, Article ID: 4186468.

https://doi.org/10.1155/2020/4186468

[18] Schiraldi, A. and Foschino, R. (2021) An Alternative Model to Infer the Growth of Psychrotrophic Pathogenic Bacteria. Journal of Applied Microbiology.

[19] Schiraldi, A. and Foschino, R. (2021) Time Scale of the Growth Progress in Bacterial 
Cultures: A Self-Consistent Choice. RAS Microbiology and Infectious Diseases, 1, 1-8.

[20] Swinnen, I.A.M., Bernaerts, K., Dens, E.J.J., Geeraerd, A.H. and Van Impe, J.F. (2004) Predictive Modelling of the Microbial Lag Phase: A Review. International Journal of Food Microbiology, 94, 137-159. https://doi.org/10.1016/j.ijfoodmicro.2004.01.006

[21] Yates, G.T. and Smotzer, T. (2007) On the Lag Phase and Initial Decline of Microbial Growth Curves. Journal of Theoretical Biology, 244, 511-517. https://doi.org/10.1016/j.jtbi.2006.08.017

[22] Baranyi, J., George, S.M. and Kutalik, Z. (2009) Parameter Estimation for the Distribution of Single Cell Lag Times. Journal of Theoretical Biology, 259, 24-30. https://doi.org/10.1016/j.jtbi.2009.03.023

[23] Bertrand, R.L. (2019) Lag Phase Is a Dynamic, Organized, Adaptive, and Evolvable Period That Prepares Bacteria for Cell Division. Journal of Bacteriology, 201, 1-21. https://doi.org/10.1128/JB.00697-18

[24] Altilia, S., Foschino, R., Grassi, S., Antoniani, D., Dal Bello, F. and Vigentini, I. (2021) Investigating the Growth Kinetics in Sourdough Microbial Associations. Food Microbiology, 99, Article ID: 103837. https://doi.org/10.1016/j.fm.2021.103837

[25] Praderio, G., Schiraldi, A., Sorlini, C., Stassi, A. and Zanardini, E. (1993) Microbiological and Calorimetric Investigations on Degraded Marbles from Cà d'Oro Facade (Venice). Thermochimica Acta, 227, 205-213. https://doi.org/10.1016/0040-6031(93)80263-A

[26] Kocharunchitt, C. and Ross, T. (2015) Challenge Studies Involving Cheese Production. Food Safety Centre, University of Tasmania, Hobart, ComBase EcR31_Feta_15C_P.

[27] Peleg, M. (2003) Microbial Survival Curves: Interpretation, Mathematical Modeling, and Utilization. Comments on Theoretical Biology, 8, 357-387.

https://doi.org/10.1080/08948550302436

[28] Salazar, J.K., Bathija, V.M., Carstens, C.K., Narula, S.S., Shazer, A., Stewart, D. and Tortorello, M.L. (2018) Listeria monocytogenes Growth Kinetics in Milkshakes Made from Naturally and Artificially Contaminated Ice Cream. Frontiers in Microbiology, 9 , 62. https://doi.org/10.3389/fmicb.2018.00062

[29] Vadasz, P. and Vadasz, A.S. (2002) The Neoclassical Theory of Population Dynamics in Spatially Homogeneous Environments. (II) Non-Monotonic Dynamics, Overshooting and Oscillations. Physica A: Statistical Mechanics and Its Applications, 309, 360-380. https://doi.org/10.1016/S0378-4371(02)00587-3

[30] Gardikis, K., Signorelli, M., Ferrario, C., Schiraldi, A., Fortina, M.G., Hatziantoniou, S., Demetzos, C. and Fessas, D. (2017) Microbial Biosensors to Monitor the Encapsulation Effectiveness of Doxorubicin in Chimeric Advanced Drug Delivery Nano Systems: A Calorimetric Approach. International Journal of Pharmaceutics, 516, 178-184. https://doi.org/10.1016/j.ijpharm.2016.11.033 